\title{
Optic coherence tomography in a patient with diffuse unilateral subacute neuroretinitis
}

\author{
Tomografia de coerência óptica em neuretinite subaguda \\ difusa unilateral
}

\author{
Alexandre Henrique Gomes ${ }^{1}$ \\ Carlos Alexandre de Amorim Garcia ${ }^{2}$ \\ Paulo de Souza Segundo ${ }^{3}$ \\ Carlos Alexandre de Amorim Garcia Filho ${ }^{4}$ \\ Ana Claudia de Amorim Garcia ${ }^{5}$
}

Study carried out at Universidade Federal do Rio Grande do Norte - UFRN - Natal (RN) - Brazil.

${ }^{1} \mathrm{MD}$ at Universidade Federal do Rio Grande do Norte UFRN - Natal (RN) - Brazil.

${ }^{2} \mathrm{MD}$ PhD at UFRN - Natal (RN) - Brazil.

${ }^{3} \mathrm{MD}$ at UFRN - Natal (RN) - Brazil.

${ }^{4} \mathrm{MD}$ at UFRN - Natal (RN) - Brazil.

${ }^{5}$ MS at UFRN - Natal (RN) - Brazil.

Address for correspondence: Carlos Alexandre de Amorim Garcia. Departamento Oftalmologia-UFRN, Hospital Universitário Onofre Lopes - Natal (RN)

CEP 59075-250

E-mail: prontoc.de.olhos@digi.com.br

Recebido para publicação em 14.12.2007

Última versão recebida em 11.12.2008

Aprovação em 08.01.2009

Nota Editorial: Depois de concluída a análise do artigo sob sigilo editorial e com a anuência da Dra. Luciana Peixoto Finamor sobre a divulgação de seu nome como revisora, agradecemos sua participação neste processo.

\begin{tabular}{|l|}
\hline ABSTRACT \\
\hline Purpose: To measure retinal nervous fiber layer (RNFL) thickness using \\
OCT3 (Carl-Zeiss) in patients with diffuse unilateral subacute neu- \\
roretinitis (DUSN) with or without live worm and correlate it with visual \\
acuity. Methods: RNFL thickness, using RNFL thickness 3.4 program \\
and best corrected visual acuity were measured in patients with DUSN \\
between January 2005 and December 2006. Results: Thirty-eight patients, \\
aged 9-42 years were selected, of whom 20 had live worm. Mean RNFL \\
was 71.55 \pm 27.26 in the DUSN eye and 103.07 \pm 20.66 in the contralateral \\
eye (p<0.001). Pearson's correlation between visual acuity and RNFL \\
was r=-0.522 (p<0.001) in the DUSN eye and r=-0.097 (p=0.509) in the \\
contralateral eye. Conclusion: RNFL thickness in DUSN patients is \\
directly proportional to visual acuity. Further research is needed to \\
reinforce the correlation between visual acuity and thickness of the nerve \\
fibers in patients with DUSN to follow them after the treatment.
\end{tabular}

Keywords: Tomography, optical coherence; Optic nerve diseases; Nerve fibers/pathology; Optic neuritis/pathology; Retinitis/pathology; Eye infections, parasitic/pathology; Diagnostic techniques, ophthalmological

Diffuse unilateral subacute neuroretinitis (DUSN) is an ocular infection disease caused by a nematode capable of causing loss of vision in one or, rarely, both eyes ${ }^{(1-4)}$. It is most frequently seen in healthy children or young adults with no significant past ocular history. It is one of the main causes of unilateral blindness in Northeast Brazil ${ }^{(5)}$. It is a diffuse uveitis characterized in the acute phase by swelling of the optic disc, recurrent crops of evanescent, multifocal, white-yellowish lesions at the outer retina and choroid level ${ }^{(6)}$. Optic atrophy and severe retinal arterial narrowing seems to define best the late stage $\mathrm{e}^{(1)}$.

Optical coherence tomography (OCT) is a noncontact, noninvasive diagnostic technique that allows measurement of retinal nerve fiber layer (RNFL) thickness by in vivo visualization of the retina and RNFL with good reproducibility ${ }^{(7)}$.

The purpose of the study is to evaluate retinal nerve fiber layer (RNFL) thickness using optic coherence tomography (OCT) in patients with DUSN with or without live worm and correlate it with visual acuity.

\section{METHODS}

Patients with unilateral DUSN diagnosis were included in this study, carried out between January, 2005 and December, 2006 at the Department 
of Ophthalmology of the Federal University of Rio Grande do Norte, Brazil. This is a prospective study.

All subjects underwent a complete ophthalmologic examination, including the best corrected visual acuity, slit lamp examination, intraocular pressure and optic nerve retinal evaluation. Any other ocular disease was considered exclusion criterion. The contralateral eye was used as control group.

Subjects underwent ocular imaging with dilated pupils using Stratus OCT (Carl-Zeiss Meditec, Dublin, California, USA). Quality scans had to have focused images, signal strength $\geq 7$, and a circular ring around the optic disc for RNFL scans. Average RNFL thickness (optic disc) protocol was used to obtain thickness measurements.

Statistical analysis was performed using Paired Student's $t$ test and Pearson's correlation test. A significance level of $5 \%$ was set for all analyses.

Informed consent was obtained from all participants and the study was approved by the institutional ethics committee.

\section{RESULTS}

A total of 38 patients, aged 9 to 42 years, were included in the study, of whom 20 had live worm. Mean RNFL was $71.55 \pm$ 27.26 in the DUSN eye and $103.07 \pm 20.66$ in the contralateral eye $(\mathrm{p}<0.001)$. Pearson's correlation between visual acuity and RNFL was $r=-0.522(\mathrm{p}<0.001)$ in the DUSN eye and $\mathrm{r}=-0.097(\mathrm{p}=0.509)$ in the contralateral eye.

The statistical analysis comparing the variables (MaxMin, Smax, Imax, Savg; Iavg, Avg thickness) between the DUSN eyes and the control group is shown in Table 1. DUSN eyes had a statistically significant decrease in RNFL when compared to the control group.

Pearson's correlation test showed a decrease in RNFL directly proportional to the decrease in visual acuity converted to $\log$ MAR, with statistical significance in the parameters MaxMin, Smax, Imax, Savg, Iavg, Avg thickness (Table 2).

\section{DISCUSSION}

Only one case of strongly suspected clinical DUSN was histopathologically studied by Gass and $\mathrm{Scelfo}^{(8)}$. The eye showed evidence of a nonspecific inflammatory process, involving the vitreous body, optic nerve, retina and choroid. Histopathologic data were not sufficient to explain visual loss in this case, which contributed to speculation about the role of functional mechanisms in causing visual damage ${ }^{(6)}$. The mechanism of this phenomenon is explained by Oréfice et al. as being a consequence of possible inflammatory and/or toxic aggression towards retinal bipolar cells ${ }^{(9)}$.

The Stratus OCT provides information on the probability of abnormal patient examination results after comparison with an internal normative database ${ }^{(10)}$.

Table 3 illustrates a number of cases that show the correlation between RNFL thickness and visual acuity.

Statistical analysis showed that there is no significant difference between RNFL thickness in patients with or without live worm. However, there is statistical significance between decreased RNFL thickness and worse visual acuity.

Additional studies are needed to validate the use of this examination in the follow-up of patients whose worm was eliminated. This is particularly important in patients whose worm was not located and who were submitted to clinical treatment, so that fiber nerve atrophy can be accompanied.

\section{RESUMO}

Objetivo: Avaliar o uso do OCT3 (Carl-Zeiss) para medir a espessura da camada de fibras nervosas em pacientes com neu-

Table 1. Statistical analysis comparing the variables (Max-Min, Smax, Imax, Savg; lavg, Avg thickness) between the DUSN eyes and the

\begin{tabular}{|c|c|c|c|c|c|}
\hline Variable & Eye & $\begin{array}{c}\text { Present }(n=17) \\
\text { Mean=DF }\end{array}$ & $\begin{array}{c}\text { Absent }(n=21) \\
\text { Mean=DF }\end{array}$ & $\begin{array}{l}\text { Total group }(n=D P) \\
\text { Mean=DF }\end{array}$ & p valor \\
\hline $\begin{array}{l}\text { Smax (superior maximum) } \\
\text { Smax (superior maximum) }\end{array}$ & $\begin{array}{l}\text { Affected } \\
\text { Healthy }\end{array}$ & $\begin{array}{l}117.71=51.32 \\
105.00=47.89\end{array}$ & $\begin{array}{l}113.82=31.03 \\
103.48=23.73\end{array}$ & $\begin{array}{l}115.46=42.95 \\
104.10=30.01\end{array}$ & $\begin{array}{l}\mathrm{P}^{(*)}=0.775 \\
\mathrm{P}^{(*)}=0.905\end{array}$ \\
\hline$p$ valor & & $P^{(*)}=0.022^{*}$ & $\mathrm{P}^{(*)}<0.001^{*}$ & $P^{(*)}<0.001^{*}$ & \\
\hline $\begin{array}{l}\text { Imax (inferior maximum } \\
\text { Imax (inferior maximum) }\end{array}$ & $\begin{array}{l}\text { Affected } \\
\text { Healthy }\end{array}$ & $\begin{array}{l}122.88=51.35 \\
172.76=57.30\end{array}$ & $\begin{array}{l}129.05=51.80 \\
170.57=26.00\end{array}$ & $\begin{array}{l}126.29=50.87 \\
174.87=42.00\end{array}$ & $\begin{array}{l}\mathrm{P}^{(*)}=0.716 \\
\mathrm{P}^{(*)}=0.004\end{array}$ \\
\hline $\mathrm{p}$ valor & & $P^{(*)}=0.016^{*}$ & $P^{(*)}=0.001^{*}$ & $P^{(*)}=0.001^{*}$ & \\
\hline $\begin{array}{l}\text { Savg (superior avg) } \\
\text { Savg (superior avg) }\end{array}$ & $\begin{array}{l}\text { Affected } \\
\text { Healthy }\end{array}$ & $\begin{array}{r}88.04=41.42 \\
129.82=30.49\end{array}$ & $\begin{aligned} 80.62 & =28.64 \\
130.70 & =20.26\end{aligned}$ & $\begin{aligned} 94.34 & =34.08 \\
130.34 & =28.25\end{aligned}$ & $\begin{array}{l}\mathrm{P}^{(*)}=0.470 \\
\mathrm{P}^{(*)}=0.925\end{array}$ \\
\hline $\mathrm{p}$ valor & & $\mathbf{P}^{(*)}=0.017^{*}$ & $P^{(*)}=0.001^{*}$ & $\mathbf{P}^{(*)}=0.001^{*}$ & \\
\hline $\begin{array}{l}\text { lavg (inferior avg) } \\
\text { lavg (inferior avg) }\end{array}$ & $\begin{array}{l}\text { Affected } \\
\text { Healthy }\end{array}$ & $\begin{array}{r}98.53=43.74 \\
131.35=38.50\end{array}$ & $\begin{aligned} 90.81 & =40.28 \\
143.29 & =21.57\end{aligned}$ & $\begin{aligned} 94.28 & =41.47 \\
137.95 & =30.47\end{aligned}$ & $\begin{array}{l}P^{(*)}=0.575 \\
P^{(*)}=0.265\end{array}$ \\
\hline $\mathrm{p}$ valor & & $P^{(*)}=0.041^{*}$ & $P^{(*)}=0.001^{*}$ & $P^{(*)}=0.001^{*}$ & \\
\hline $\begin{array}{l}\text { Avg.Thick (avg. thickness) } \\
\text { Avg.Thick (avg. thickness) }\end{array}$ & $\begin{array}{l}\text { Affected } \\
\text { Healthy }\end{array}$ & $\begin{array}{l}74.23=29.77 \\
98.47=27.34\end{array}$ & $\begin{aligned} 89.39 & =25.59 \\
106.80 & =12.82\end{aligned}$ & $\begin{aligned} 71.55 & =27.28 \\
103.07 & =20.60\end{aligned}$ & $\begin{array}{l}\mathrm{P}^{(*)}=0.593 \\
\mathrm{P}^{(*)}=0.258\end{array}$ \\
\hline $\mathrm{p}$ valor & & $P^{(*)}=0.043^{*}$ & $P^{(*)}=0.001^{*}$ & $P^{(*)}=0.001^{*}$ & \\
\hline
\end{tabular}




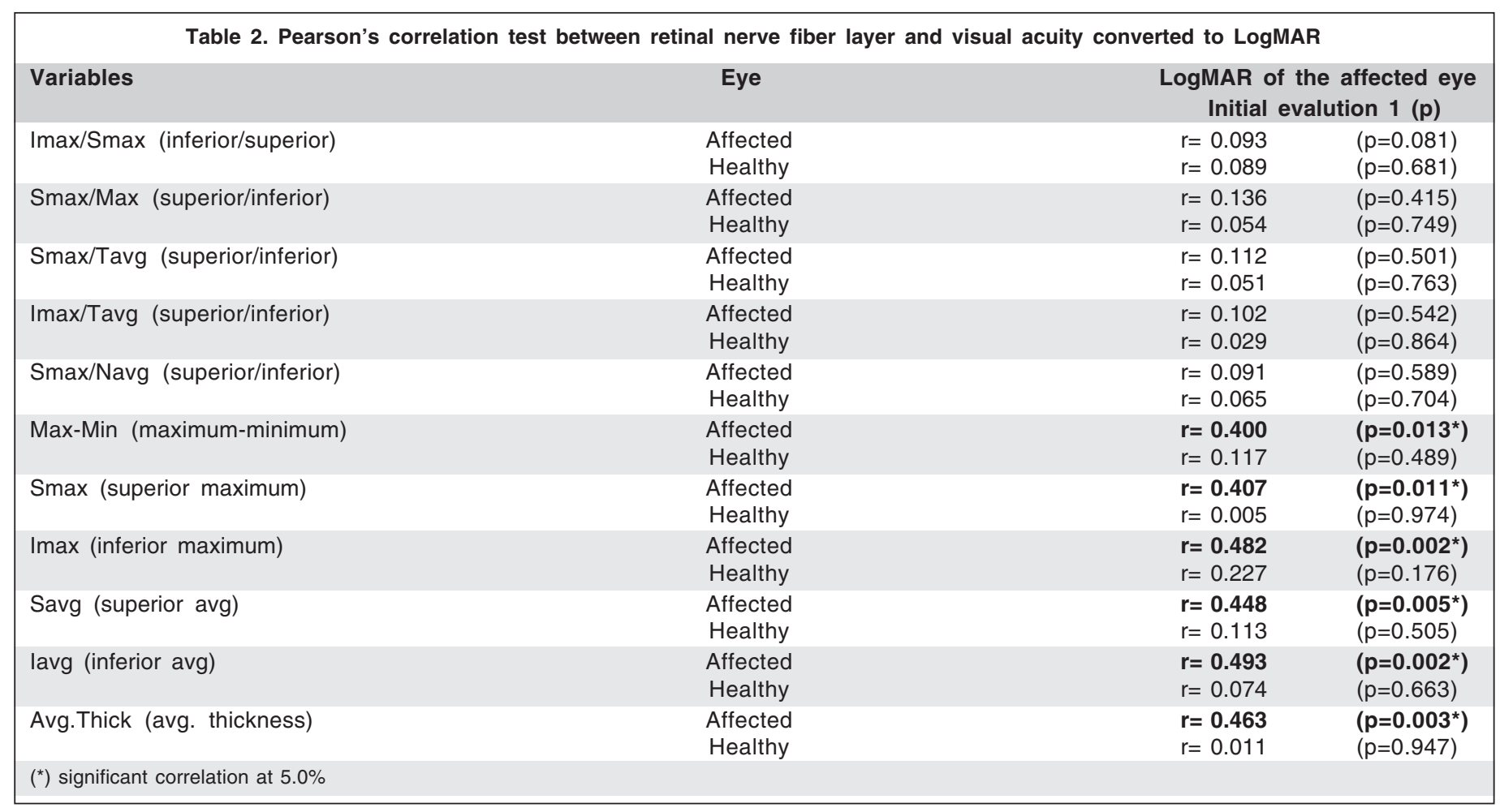

\begin{tabular}{|lccc|}
\hline \multicolumn{4}{|c|}{ Table 3. Correlation between retinal nerve fiber layer and visual acuity } \\
Visual acuity & $\begin{array}{c}\text { Thickness } \\
\text { avg. }\end{array}$ & Visual acuity & $\begin{array}{c}\text { Thickness } \\
\text { avg. }\end{array}$ \\
HM & 32.19 & CF & 56.33 \\
HM & 31.09 & CF & 54.13 \\
HM & 35.58 & CF & 66.54 \\
HM & 70.98 & $20 / 400$ & 67.23 \\
HM & 72.60 & $20 / 400$ & 89.43 \\
CF & 55.10 & $20 / 400$ & 91.51 \\
CF & 49.12 & $20 / 400$ & 56.11 \\
CF & 30.53 & $20 / 200$ & 80.21 \\
CF & 89.92 & $20 / 200$ & 69.48 \\
CF & 55.23 & $20 / 200$ & 48.52 \\
CF & 43.77 & $20 / 200$ & 92.36 \\
CF & 56.05 & $20 / 100$ & 95.13 \\
CF & 55.24 & $20 / 50$ & 90.09 \\
CF & 67.65 & $20 / 50$ & 93.80 \\
CF & 35.27 & $20 / 40$ & 106.42 \\
\hline
\end{tabular}

roretinite unilateral subaguda difusa (DUSN) e correlacionar com a acuidade visual. Métodos: Foi medido a espessura da camada de fibras nervosas, utilizando programa "RNFL thickness 3.4" e a melhor acuidade visual de pacientes com DUSN entre janeiro de 2005 e dezembro 2006. Resultados: Trinta e oito pacientes, com idade entre 9-42 anos foram selecionados para este estudo, sendo que 20 casos apresentavam larva viva localizada. A média da RNFL foi 71,55 $\pm 27,26$ nos olhos com DUSN e 103,07 $\pm 20,66$ nos olhos contralaterais ( $p<0,001)$. Correlação de Pearson entre a acuidade visual e a espessura da camada de fibras nervosas foi $r=-0,522(p<0,001)$ nos olhos com DUSN e $r=-0,097(p=0,509)$ nos olhos contralaterais. Conclusão: A espessura da camada de fibras nervosas de pacientes com DUSN apresenta uma correlação diretamente proporcional com a acuidade visual. Novos estudos são necessários para reforçar a correlação entre a acuidade visual e a espessura da camada de fibras nervosas nos pacientes com DUSN com a finalidade de acompanhar os pacientes após o tratamento.

Descritores: Tomografia de coerência óptica; Doenças do nervo óptico; Fibras nervosas/patologia; Neurite óptica/patologia; Retinite/patologia; Infecções oculares parasitárias; Técnicas de diagnóstico oftalmológico

\section{REFERENCES}

1. Gass JD, Braunstein RA. Further observations concerning the diffuse unilateral subacute neuroretinitis syndrome. Arch Ophthalmol. 1983;101(11):1689-97.

2. Goldberg MA, Kazacos KR, Boyce WM, Ai E, Katz B. Diffuse unilateral subacute neuroretinitis. Morphometric, serologic, and epidemiologic support for Baylisascaris as a causative agent. Ophthalmology. 1993;100(11):1695-701. Comment in: Ophthalmology. 1994;101(6):971-2.

3. Gass JDM. Diffuse unilateral subacute neuroretinitis. In: Gass JDM, editor. Stereoscopic atlas of macular diseases: diagnosis and treatment. $4^{\text {th }}$ ed. St. Louis: Mosby-Year Book Inc.; 1997. p.622-8.

4. de Souza EC, Abujamra S, Nakashima Y, Gass JD. Diffuse bilateral subacute neuroretinitis: first patient with documented nematodes in both eyes. Arch Ophthalmol. 1999;117(10):1349-51.

5. Garcia CAA, Gomes AHB, Barbosa MFA, Rocha MLR, Uchôa RAC. Aspectos clínicos e epidemiológicos das uveítes em Natal - RN. Rev Bras Oftalmol. 2002;61(2):121-9.

6. Oréfice F, Garcia CA, Paranhos FR. Neuroretinite subaguda unilateral difusa (DUSN). In: Oréfice F, editor. Uveíte clínica e cirúrgica. $2^{\underline{a}}$ ed. Rio de Janeiro: Cultura Médica; 2005. v. 2. p.885-916.

7. Sihota R, Sony P, Gupta V, Dada T, Singh R. Diagnostic capability of optical 
coherence tomography in evaluating the degree of glaucomatous retinal nerve fiber damage. Invest Ophthalmol Vis Sci. 2006;47(5):2006-10.

8. Gass JD, Scelfo R. Diffuse unilateral subacute neuroretinitis. J R Soc Med. 1978;71(2):95-111.

9. Oréfice F, Gonçalves ER, Siqueira RC, Nehemy MB. Estudo de 21 casos de neuroretinite subaguda unilateral difusa (DUSN): dois casos com larva móvel sub-retiniana. Rev Bras Oftalmol. 1994;53(6):23-45.

10. Monteiro ML, Moura FC, Medeiros FA. Diagnostic ability of optical coherence tomography with a normative database to detect band atrophy of the optic nerve. Am J Ophthalmol. 2007;143(5):896-9. 